\title{
An Iterative Refining Mechanism for General Job Shop Scheduling Problems
}

\author{
Tsung-Che Chiang \\ Dept. of Computer Science and Information Engineering \\ National Taiwan University \\ No.1, Sec. 4, Roosevelt Road, Taipei, Taiwan \\ tcchiang@ieee.org
}

\author{
Li-Chen Fu \\ Dept. of Computer Science and Information Engineering \\ National Taiwan University \\ No.1, Sec. 4, Roosevelt Road, Taipei, Taiwan \\ lichen@ntu.edu.tw
}

\begin{abstract}
This paper addresses the general job shop scheduling problem with the objective as how to increase the meet-due-date rate. An iterative schedule refining mechanism is proposed, which iteratively adjusts the estimation of the remaining processing times of jobs in a dynamic and stage-specific manner. The refining mechanism is applied to a recently proposed sequencing heuristic and the integrated approach shows satisfactory performance through a comprehensive simulation study. Besides, we also show that the proposed refining mechanism can bring much improvement to several conventional rules.
\end{abstract}

Index Terms - Scheduling; Job shops; Heuristics;

\section{INTRODUCTION}

Job shop scheduling is essentially a task of allocation of machines over time to the jobs in such a way that the selected performance criteria are optimized. It is usually reduced to a sequencing problem - to determine the sequence of jobs to be processed on machines. In this paper, the performance measure is the meet-due-date rate, reflecting the recent emphasis given to customer satisfaction in the industry [15].

Among the approaches to solve this problem, sequencing rules (or scheduling heuristics) are pervasively used in the industry $[8,10,16]$ because of their satisfactory performance, low computation requirements, ease of implementation, intuitive appeal, and flexibility to incorporate domain knowledge and expertise. Generally, sequencing rules give priority values to jobs when a selection is to be made, and the sequence of processing order of jobs is determined based on these priority values. Many conventional sequencing rules, like the shortest processing time (SPT) rule, can be found in the survey and simulation-based reports by Wein [1], Chang [6], Sabuncuoglu [13] and Costa et al. [14]. In particular, Lu et al. [5] used a class of least slack-based rules to reduce the mean and variance of cycle time in semiconductor manufacturing plants, and Li et al. [7] proposed the minimum inventory variability scheduling rule to achieve the same goal as Lu et al. did. Kim et al. [18] proposed several due-date based rules for lot release control, lot scheduling, and batch scheduling. An interactive scheduler built by Huang et al. [11] took sequencing rules as a major component in the knowledge base. Besides the efforts to the invention of efficient heuristics, there were also research works on combining the existing rules $[19,20]$ and dynamic rule selection [17] to solve the production scheduling problems.
The remaining part of this paper is organized as follows: in Section II, we give a review of a recently developed sequencing rule, the Enhanced Critical Ratio (ECR) heuristic [21]. Then, the iterative schedule refining mechanism is proposed in Section III. A study of the performance of the proposed ideas is given in Section IV, and finally conclusions are made in Section V.

\section{THE ECR SEQUENCING HeURISTIC}

As mentioned, many sequencing rules have been proposed in the literature. In our observation, they all share a common feature - when applying these rules to do job sequencing, only information concerning an individual job is used. For example, the SPT rule prioritizes jobs by their processing time and the earliest-due-date (EDD) rule prioritizes jobs by their due dates. It can be expected that they can not provide good decisions with this small amount of information. In our solution, we proposed a new sequencing rule, the enhanced critical ratio (ECR) rule, which takes into account the "group information" based on the concept of the critical ratio (CR) rule. The basic idea of ECR rule is to pick a job as the next processing target such that the urgency of all jobs is kept minimal after that job is processed. The detailed steps to apply the ECR rule and the preliminary experimental results were presented in [21].

\section{THE ITERATIVE SCHEDULE REFINING MECHANISM}

The remaining processing time is a factor commonly adopted in the design of sequencing rules. It is usually calculated as the sum of processing times of unfinished operations. However, this sum (hereafter we call it the minimal remaining processing time, $r p t_{\text {minimal }}$ ) is only a lower bound and the actual remaining processing time is usually longer than $r p t_{\text {minimal }}$ because of resource contention in the plant. To cope with this problem, a common solution in the literature estimates the actual remaining processing time by multiplying an amplification ratio $(a)$ to the minimal remaining processing time excluding the processing time of current operation $\left(p_{c}\right)$, i.e., $r p t=p_{c}+a \times\left(r p t_{\text {minimal }}-p_{c}\right)$ where the value of $a$ is determined empirically. This solution is static since the amplification of $r p t_{\text {minimal }}$ in the estimate is assumed in a uniform way regardless of the difference of degree of contention with respect to different job types and the stages at which the jobs are staying. 


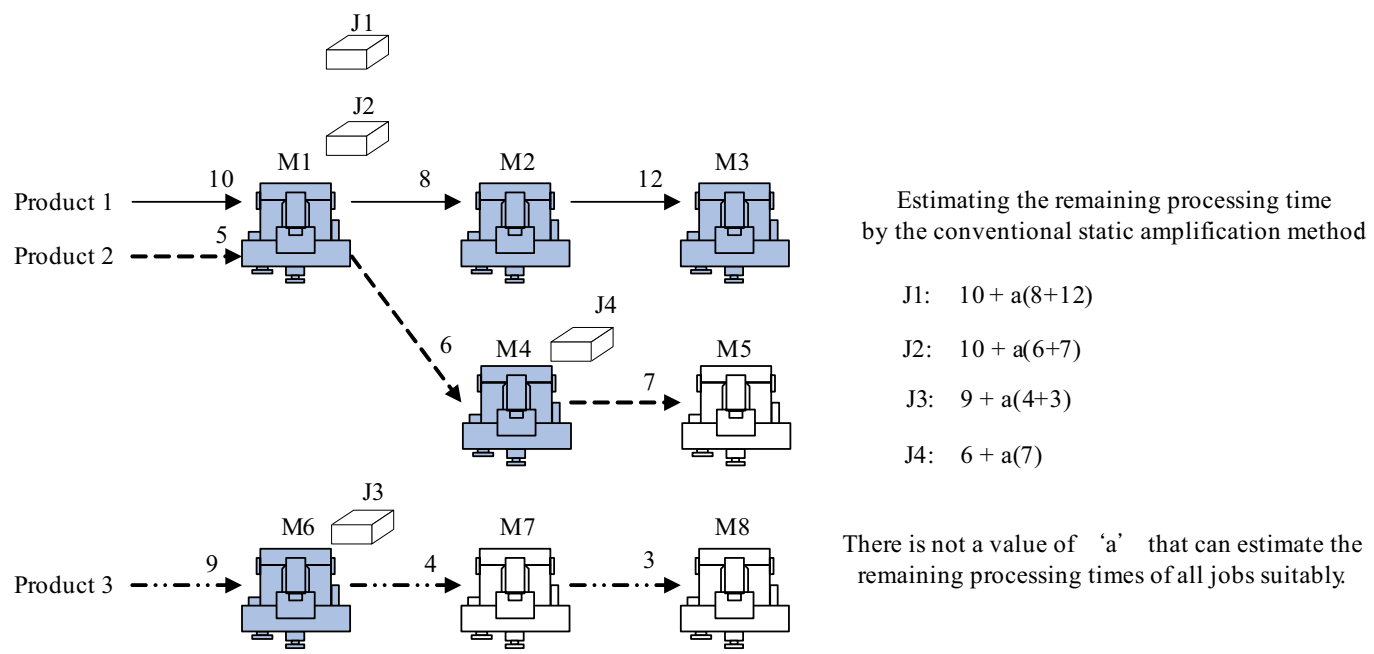

Fig. 1 An example for the drawbacks of the conventional method to estimate the remaining processing time

To realize the drawbacks of the static amplification method, we take Fig. 1 as an example. In this plant, there are many kinds of products and the routes of three of them are depicted. The number above the arrow is the processing time on the machines for each product; for example, the processing time for M1 to process jobs of product 1 is 10. Severe resource contention takes place on the colored machines (M1, M2, M3, M4, and M6). The other three machines are dedicated to only one kind of product. For the job of product 1 , J1, the amplification ratio should be large to reflect the fierce resource contention occurred on machines M2 and M3. Nevertheless, for the job of product $3, \mathrm{~J} 3$, the amplification ratio should be close to 1 since machines $\mathrm{M} 7$ and M8 are dedicated to product 3 . In this case, the conventional static amplification method, which estimates the actual remaining processing time by amplifying $r p t_{\text {minimal }}$ in a uniform way for all kinds of products, is believed to fail.

Even for jobs of the same product, the conventional method can also have problems. Given two jobs of product 2 , one $(\mathrm{J} 2)$ is at machine M1 and the other (J4) is at M4. The actual remaining processing time of $\mathrm{J} 2$ should be significantly different from its $r p t_{\text {minimal }}$ whereas the actual and minimal remaining processing times of $\mathrm{J} 4$ should be almost the same. Again a single value of the amplification ratio $a$ can not satisfy this situation. Besides, amplifying the processing time on M5 for J2 is also unreasonable because there is no resource contention on M5.

After realizing the drawbacks of the conventional static amplification method, we propose a schedule refining mechanism that has two features:

1. It considers the degree of contention of machines in a job-specific and operation-specific manner. The remaining processing time is estimated according to the actual queueing time of each job at each operation.

2. It refines the schedule iteratively based on the estimates of remaining processing times in each iteration.

The solution based on the ECR heuristic and the iterative refining mechanism is named I-ECR. The entire procedure of I-ECR is given as follows:

\section{$\underline{\text { Notations }}$}

$p_{i j} \quad$ the processing time required by the $j^{\text {th }}$ operation of job $i$

$p_{i j}^{\prime} \quad$ the predicted processing time required to finish the $j^{\text {th }}$ operation of job $i$

$q_{i j} \quad$ the queueing time of job $i$ for processing the $j^{\text {th }}$ operation, i.e., the duration from the instant when the $(j-1)^{\text {th }}$ operation was completed to the instant when the $j^{\text {th }}$ operation started

$r_{i} \quad$ the number of operations of job $i$

$r p t_{i j}$ the estimated remaining processing time of job $i$ which is waiting for processing of the $j^{\text {th }}$ operation

$n \quad$ the number of jobs

$t \quad$ the iteration count

$Q \quad$ the amplification ratio of queueing time, a parameter of the refining mechanism

$T \quad$ the number of iterations for schedule refining, also a parameter of the refining mechanism

Step0. Preparation:

$$
\begin{aligned}
q_{i j} & =0 \quad \forall i=1 \ldots n, \quad j=1 \ldots r_{i} . \\
t & =1 .
\end{aligned}
$$

Step1. Updating remaining processing times:

$$
\begin{aligned}
r p t_{i j} & =p_{i j}+\sum_{k=j+1}^{r_{i}} p_{i k}^{\prime}=p_{i j}+\sum_{k=j+1}^{r_{i}}\left(p_{i k}+Q \cdot q_{i k}\right) \\
& =r p t_{\text {minimal }}+Q \cdot \sum_{k=j+1}^{r_{i}} q_{i k} .
\end{aligned}
$$

Step2. Run a simulation and apply the ECR heuristic (described in Section II) to make a schedule $S_{t}$.

Step3. Renew queueing times $q_{i j}$ based on the schedule $S_{t}$ obtained in Step2.

Step4. $t=t+1$. If $t \leq T$, goto Step1.

Otherwise, the refining process ends, and the best schedule among all $T$ schedules is reported. 


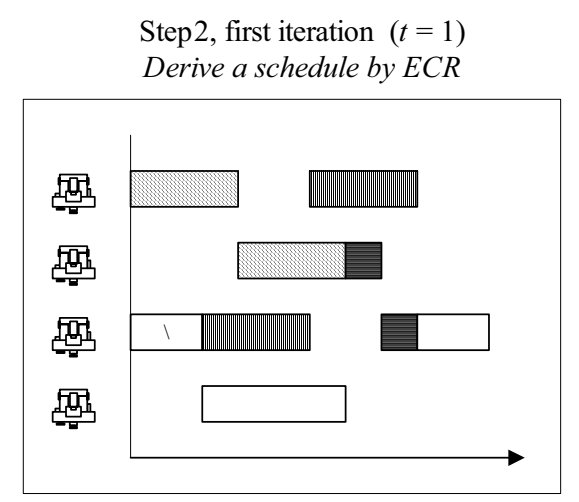

Step3, first iteration $(t=1)$

Renew queueing times

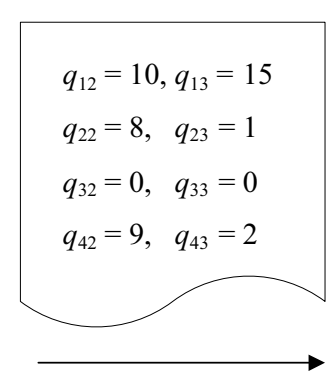

Step1, second iteration $(t=2)$

Update remaining processing times

$$
\begin{aligned}
& r p t_{11}=(10+8+12)+Q(10+15) \\
& r p t_{21}=(10+6+7)+Q(8+1) \\
& r p t_{31}=(9+4+3)+Q(0+0) \\
& r p t_{42}=(6+7)+Q(2)
\end{aligned}
$$

Fig.2 An example for the execution of the iterative refining mechanism

In this approach, the increment of remaining processing time is determined according to dynamic information obtained during the iterative refining process. The queueing time of each job at each operation is taken into account to adjust the remaining processing time.

Following the example in Fig. 1, now we use Fig. 2 to exemplify how the iterative refining mechanism works. At Step2 in the first iteration $(t=1)$, a schedule is derived by the ECR rule. Currently the remaining processing times of jobs are the minimal remaining processing times. Then we calculate queueing times $q_{i j}$ for each job at each operation. Proceeding to Step 1 in the next iteration $(t=2)$, the remaining processing times are updated based on $q_{i j}$ obtained from the previous iteration. For the case in Fig. 2, the remaining processing time for job $\mathrm{J} 1$ will receive a great increment $(Q \cdot(10+15))$ since it had a long queueing time at machines M2 and M3 (in the schedule at the first iteration). On the other hand, the remaining processing time for $\mathrm{J} 3$ is kept the same as the $r p t_{\text {minimal }}$ value because it encountered no delay at the successive operations. In this way, the remaining processing times can be adjusted in a job-specific and operation-specific manner, not be blindly amplified in a uniform way. The problem faced by the conventional static amplification method is solved.

Not only the ECR heuristic, this refining mechanism can also be applied to other heuristics. The values of parameters $Q$ and $T$ certainly have influence on the performance of this mechanism. Related experiments and discussions are provided in Section IV-C.

\section{EXPERIMENTAL RESULTS}

\section{A. Generation of testing instances}

In our experiments, testing instances are created by six parameters - number of routes $\left(N_{r}\right)$, number of stages for each route $\left(N_{s}\right)$, number of stations $\left(N_{e}\right)$, processing times $(P)$, setup times $(S)$ and due dates for each job $(D)$. The number of jobs in each instance is fixed as one hundred. There are thirteen classes of testing instances and each class contains ten instances. There is a basic class and other twelve classes are different from the basic one only by one parameter. Values of parameters of the basic class are listed in Table I and the differences of other twelve ones are listed in Table II.
TABLE I

PARAMETERS OF THE BASIC CLASS

\begin{tabular}{cccc}
\hline Parameter & Value (Range) & Parameter & Value (Range) \\
\hline$N_{r}$ & 10 & $P$ & $0.5-5$ \\
$N_{s}$ & 8 & $S$ & $0.5-2$ \\
$N_{e}$ & 25 & $D$ & $4-9$ \\
\hline
\end{tabular}

TABLE II

PARAMETERS OF OTHER TWELVE Classes

\begin{tabular}{cccccc}
\hline $\begin{array}{c}\text { Class } \\
\text { Id }\end{array}$ & $\begin{array}{c}\text { Changed } \\
\text { Parameter }\end{array}$ & Value & $\begin{array}{c}\text { Class } \\
\text { Id }\end{array}$ & $\begin{array}{c}\text { Changed } \\
\text { Parameter }\end{array}$ & Value \\
\hline 2 & $N_{r}$ & 5 & 8 & $P$ & $0.5-3$ \\
3 & $N_{r}$ & 20 & 9 & $P$ & $0.5-7$ \\
4 & $N_{s}$ & 6 & 10 & $S$ & $0.5-1$ \\
5 & $N_{s}$ & 10 & 11 & $S$ & $0.5-3$ \\
6 & $N_{e}$ & 20 & 12 & $D$ & $3-8$ \\
7 & $N_{e}$ & 30 & 13 & $D$ & $5-10$ \\
\hline
\end{tabular}

We take the basic class as an example to explain the generation of testing instances: There are 10 different routes, each consisting of 8 operations. The capabilities to perform these 80 kinds of operations are uniformly distributed to 25 stations. For each operation, only one station has the capability to perform it. The processing time of each operation is uniformly distributed over the interval $[0.5,5]$ with granularity 0.5 . The sequence dependent setup time is uniformly distributed over the interval $[0.5,2]$ with granularity 0.5 . The due date of each job is $d \times p$ where $p$ is the minimal total processing time and $d$ is a value uniformly distributed over the interval $[4,9]$ with granularity 0.1 .

The simulation engine was written in $\mathrm{C}++$ language by Microsoft Visual $\mathrm{C}++$ 6.0. All experiments were conducted on personal computers with $1 \mathrm{G} \mathrm{Hz}$ Athlon $\mathrm{CPU}$ and $256 \mathrm{MB}$ RAM.

\section{B. Performance of ECR}

\section{B.1 Benchmarks}

In order to test the performance of the ECR heuristic, we take other ten popular rules $[6,12]$ as benchmarks. Rules SPT, EDD, LTWK, SRPT, and SPT/TWK were the best five rules among forty-two rules in terms of meet-due-date rates in Chang et al. [6]. Rules CR, ATC, MDD, and COVERT were 
the best four in Kim et al.' survey [14]. These rules were also pervasively adopted as benchmarks in the literature $[3,4,13$, 14]. Thus, these rules can be representative benchmarks to verify the performance of the proposed heuristic. We tested five values for each parameter in ATC and COVERT rules, and picked the best one. The values of parameters $b$ and $k$ in ATC are one and five, and the product of parameters $b$ and $k$ in COVERT is five. The ECR heuristic takes the default setting, namely, the filtering preprocess is not used yet. Definitions of these benchmark rules can be found in $[6,12]$.

\section{B.2 Performance of each heuristic}

After conducting experiments on the 130 testing instances, we calculate the average meet-due-date rates of each testing class for each rule. Fig. 3 shows the relative performance of these rules to the best solution. For the sake of clarity, data of rules with similar performance will not be plotted in Fig. 3 and the following figures.

From the experimental results, the ECR rule performs the best in all thirteen classes. The MDD rule and SRPT rule have close performance, and are ranked the second and the third. The ATC rule is at the fourth position, followed by the EDD and COVERT rules. The last five rules are SPT, LTWK, SLACK, SPT/TWK, and CR. On average, the second best rule, MDD, reaches $80 \%$ meet-due-date rates of the ECR rule and the worst one, CR, only reaches around $60 \%$.

To see the relative performance in greater depth, we calculate the normalized value of the average meet-due-date rate by $V=\left(R_{\mathrm{i}}-R_{\min }\right) /\left(R_{\max }-R_{\text {min }}\right)$ where $R_{i}$ is the average meet-due-date rate of a rule $i$, and $R_{\min }$ and $R_{\max }$ are the minimal and maximal average meet-due-date rates among all rules applied to a certain testing class. The normalized values are shown in Fig. 4. It reveals that most benchmark rules have the normalized value well below 0.5 except the MDD heuristic. The advantage of the ECR rule is verified again by this performance gap. The computation time for running a simulation on one testing instance with each of these eleven rules is less than half second.

\section{Performance of I-ECR}

In this subsection, we will examine the benefit of our proposed iterative schedule refining mechanism. As mentioned, there are two variables which control the execution of the refining process $-Q$, which decides the percentage of queueing time being added to the predicted processing time, and $T$, which decides the number of iterations to do the refining actions. In the experiments, we use $\{0.1,0.2, \ldots 1.0\}$ as the value of $Q$ and the value of $T$ is ten. The maximal meet-due-date rate among the $100(10 \times 10)$ schedules is recorded. Only two rules including EDD and SPT are not taken into consideration since they are not affected by adjusting the predicted processing times. To denote a rule incorporated with the iterative refining mechanism, we add a prefix I-, for example, we will use I-ECR to refer to ECR with the iterative refining mechanism.

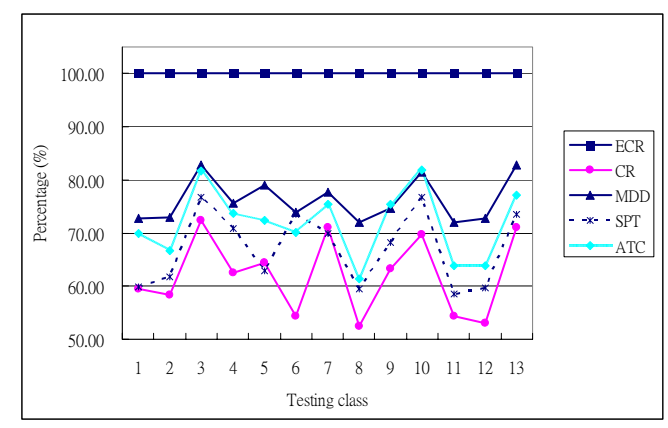

Fig. 3 The relative performance to the best heuristic for each testing class

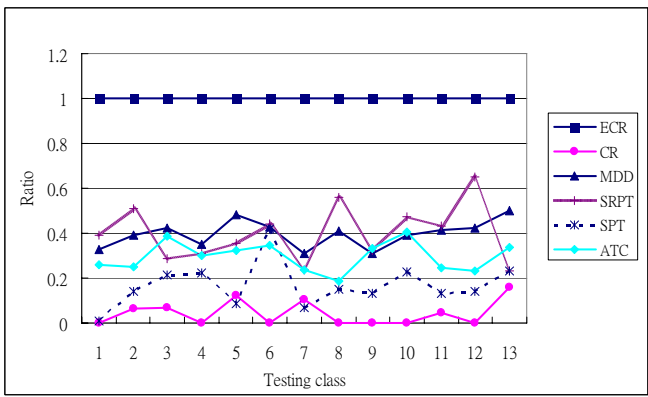

Fig. 4 The normalized performance between the best and worst heuristics

\section{C.1 Performance of each I-heuristic}

Average meet-due-date rates of each I-heuristic are summarized in Fig. 5. Again, data of rules with close values are not shown in Fig. 5 for clarity.

The I-ECR heuristic is still the best in all testing classes. The I-MDD heuristic ranks the second and its performance is only comparable to the ECR heuristic. I-SRPT, I-LTWK, and I-SPT/TWK have close performance (I-SRPT slightly better than the other two) and follow the I-MDD heuristic. The I-ATC and I-COVERT heuristics fall into the fourth group. The worst two are the I-SLACK and I-CR heuristics. The ranks of these I-heuristics are similar to the results of their counterparts without refining, except that the I-SPT/TWK and I-LTWK heuristics are now better than I-ATC because of receiving much benefit from the refining mechanism.

Referring to Fig. 6, the second best heuristic, I-MDD, approximately reaches $85 \%$ meet-due-date rates of the I-ECR heuristic on average. The worst one, I-CR, only reaches around $60 \%$.

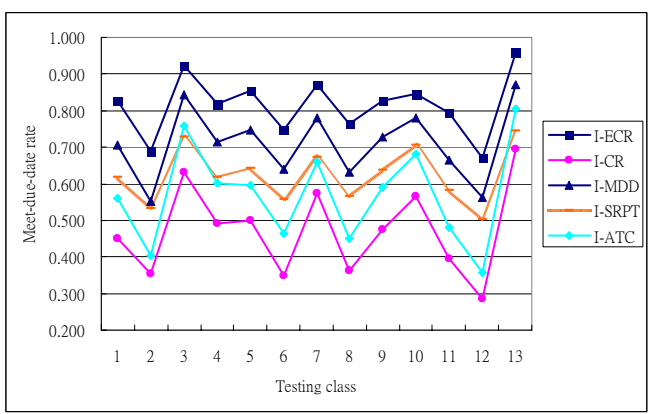

Fig. 5 Average meet-due-date rates for each I-heuristic 


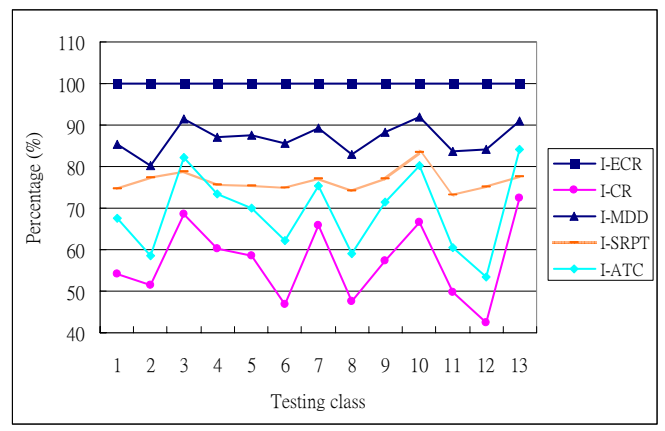

Fig. 6 The relative performance to the best I-heuristic

\section{C.2 Effects of the iterative refining mechanism}

To see the benefits brought by the refining mechanism, in Fig. 7 we show the average improvement percentage of each I-heuristic for each testing class comparing to its counterpart without iterative refining.

As can be seen in this figure, the SPT/TWK rule gets the largest gain from the refining mechanism. In our observation, the main reason is that the SPT/TWK rule has low meet-due-date rates for some problem instances and the iterative refining mechanism brings very large improvement percentage (even larger than $200 \%$ ) to these instances. The average improvement percentage in each class ranges from $12.34 \%$ to $89.22 \%$, and the average over thirteen classes is $36.32 \%$. Although the improvement is not stable, roughly speaking, SPT/TWK is highly improved by the proposed refining mechanism (so that I-SPT/TWK outperforms I-ATC now). Similar conclusion can be drawn from data of the I-LTWK heuristic.

In addition to SPT/TWK and LTWK heuristics, the MDD heuristic also gets high improvement percentage. The performance is improved for each of the 130 testing instances. It also reveals that the MDD rule, which is a mixture of EDD and SRPT rules, can perform well if the predicted processing times are set properly. The I-ECR heuristic has relatively low improvement percentage since the ECR rule has already achieved good performance. Among nine rules to be refined, the SLACK and CR rules receive the least benefit from the mechanism.

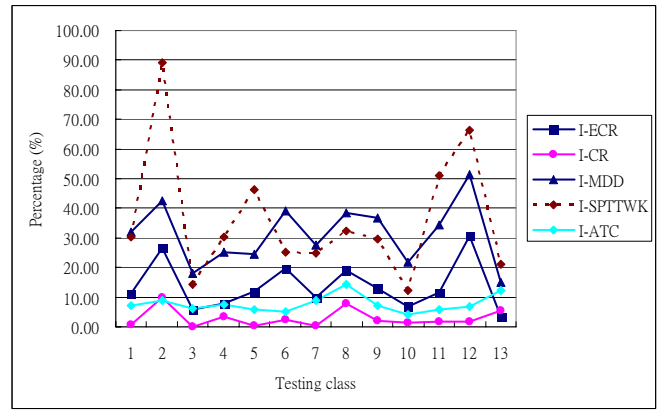

Fig. 7 Average improvement percentage by the proposed refining mechanism for each testing class

\section{C.3 Advantage of the iterative refining mechanism}

As mentioned in Section III, the traditional method to estimate the remaining processing time is to multiply a fixed amplification ratio $(a)$ to the minimal remaining processing time excluding the processing time of current operation, i.e. $r p t=p_{c}+a \times\left(r p t_{\text {minimal }}-p_{c}\right)$. To verify the performance of the proposed iterative refining mechanism, we tested the traditional method by applying it to the same nine rules, and the value of the amplification rate $a$ has the range from 1 to 10 with granularity 0.1 . In other words, we solved each testing instance for 100 times, each time with a different value of $a$. The number of schedules generated in this testing, 100, is the same as what we have on testing the proposed refining mechanism. The average improvement percentage over 130 testing instances is shown in Fig. 8. The proposed refining mechanism is denoted by I-heuristic and the traditional static amplification method is denoted by s-heuristic.

In Fig. 8, we first see an obvious advantage of the proposed mechanism over the traditional method - the traditional method does no improvement on the LTWK and SPT/TWK heuristics since the processing sequences of jobs derived by these two heuristics are not changed by static amplification of processing times. On the other hand, the proposed mechanism provides $27 \%$ and $36 \%$ improvement percentage to these two heuristics averagely. Besides, it also raised the performance of ECR, MDD, SRPT, and COVERT by more than $10 \%$. For heuristics except SPT/TWK and LTWK, heuristics including ECR, MDD, and SRPT prefer the proposed mechanism while CR, SLACK, ATC, and COVERT prefer the traditional one. The later four heuristics get relatively less improvement by the proposed mechanism because they always pursue the jobs with high degree of urgency regardless of the influence on the performance of the overall schedule. During the execution of the proposed mechanism, a tardy job in the previous iteration will have much longer remaining processing times in the next iteration, and then forms a higher pressure to those four "critical ratio-based" and "slack-based" heuristics. When pursuing these tardy jobs, more jobs might become tardy and initiates a "vicious cycle". Note that the ECR heuristic is also "critical ratio-based," but its design can eliminate this defect. Ranked by the average meet-due-date rates over thirteen classes, the best five among eighteen (9+9) I-heuristics and s-heuristics are I-ECR, s-ECR, I-MDD, s-MDD, and I-SRPT.

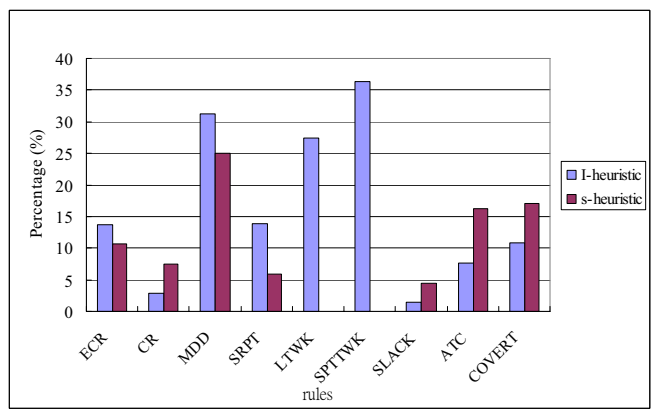

Fig. 8 Average improvement percentage over 130 instances by two refining mechanisms 


\section{4 Values of $Q$ and $T$}

In the experiments of examining the refining mechanism, we observed the occurrences of the best solutions among one hundred schedules (ten values of $Q$ by ten iterations) for each heuristic. We found that the I-ECR heuristic has a very good feature - almost all best solutions occur with $Q$ smaller than 0.5. Readers can refer to Fig. 9 to see the distributions of occurrences of best solutions by using I-ECR. It means that we can focus on a much smaller range of $Q$ and get almost the same benefit from the refining mechanism. Comparisons of the performance of the original and the compacted refining mechanism are given in Table III. It shows that we can get close performance with much reduction of computation time by limiting $Q$ in $\{0.1,0.2,0.3\}$ and $T$ to be 3 .

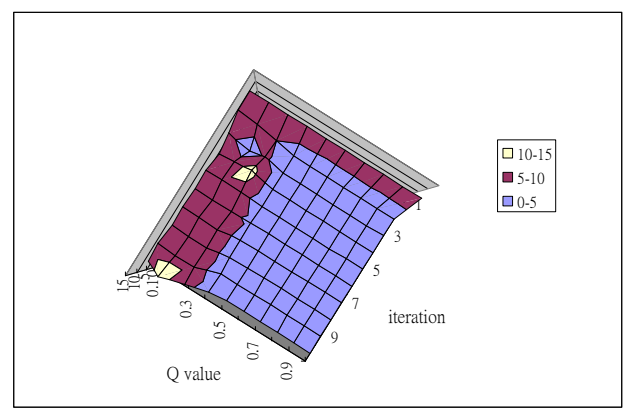

Fig. 9 Distribution of occurrences of best solutions among 130 testing instances with I-ECR w.r.t. $Q$ and iteration

TABLE III

THE PERFoRMANCE OF COMPACTED I-ECR HeURISTIC

\begin{tabular}{|c|c|c|c|c|}
\hline $\begin{array}{l}Q \\
T\end{array}$ & $\begin{array}{c}\{0.1, \ldots 1.0\} \\
10\end{array}$ & $\begin{array}{c}\{0.1, \ldots 0.3\} \\
10\end{array}$ & $\begin{array}{c}\{0.1, \ldots 0.3\} \\
5\end{array}$ & $\begin{array}{c}\{0.1, \ldots 0.3\} \\
3\end{array}$ \\
\hline \multicolumn{5}{|c|}{ Average meet-due-date rates of 130 instances } \\
\hline & 0.815 & 0.812 & 0.805 & 0.797 \\
\hline \multicolumn{5}{|c|}{ Average computation time (seconds) } \\
\hline & 30 & 9 & 4.5 & 2.7 \\
\hline
\end{tabular}

\section{CONCLUSIONS}

To increase meet-due-date rates in general job shop scheduling problems, a two-level approach is proposed. The first level is a sequencing heuristic and the second level improves the performance by an iterative refining mechanism. The careful use of critical-ratio values and its key factor remaining processing time contributes to the high performance of this approach. With gradually-enhanced relationship, users can implement and apply the sequencing heuristic at first. A considerable benefit from the scheduler can be obtained in the early construction. The extension cost to the second level of the scheduler is small while the improvement can be more than $10 \%$. For users who have difficulty to implement a new rule, the proposed refining mechanism, which is shown to be able to significantly improve several conventional rules, is recommended with few implementation efforts.

\section{ACKNOWLEDGEMENT}

This research was supported by the National Science Council of Taiwan, R.O.C., under grant number NSC932752-E-002-007-PAE.

\section{REFERENCES}

[1] Lawrence M. Wein, Scheduling semiconductor wafer fabrication, IEEE Trans. on Semiconductor Manufacturing, vol. 1, no. 3, pp. $115-130$, 1988.

[2] J. Chandra, J. Talavage, Intelligent dispatching for flexible manufacturing, International Journal of Production Research, vol. 29, no. 11 , pp. $2259-2278,1991$.

[3] Min Hee Kim, Yeong-Dae Kim, Simulation-based real-time scheduling in a flexible manufacturing system, Journal of Manufacturing Systems, vol. 13 , no. 2 , pp. $85-93,1994$.

[4] Irfan M. Ovacik, Reha Uzsoy, Exploiting shop floor status information to schedule complex job shops, Journal of Manufacturing Systems, vol. 13, no. 2 , pp. $73-84,1994$.

[5] Steve C. H. Lu, Deepa Ramaswamy, P. R. Kumar, Efficient scheduling policies to reduce mean and variance of cycle-time in semiconductor manufacturing plants, IEEE Trans. on Semiconductor Manufacturing, vol. 7 , no. 3 , pp. $374-388,1994$.

[6] Yih-Long Chang, Toshiyuki Sueyoshi, Robert S. Sullivan, Ranking dispatching rules by data envelopment analysis in a job shop environment, IIE Transactions, vol. 28, pp. 631-642, 1996.

[7] Shu Li, Tom Tang, Donald W. Collins, Minimum inventory variability schedule with applications in semiconductor fabrication, IEEE Trans. on Semiconductor Manufacturing, vol. 9, no. 1, pp. 145 - 149, 1996.

[8] K. Appleton-Day, L. Shao, Real-time dispatch gets real-time results in AMD's Fab25, Proc. of IEEE/SEMI Advanced Semiconductor Manufacturing Conference and Workshop, pp. 444 - 447, 1997.

[9] Young Hoon Lee, Kumar Bhaskaran, Michael Pinedo, A heuristic to minimize the total weighted tardiness with sequence-dependent setups, IIE Transaction, vol. 29, no. 1, pp. 45 - 52, 1997.

[10]A. Giegandt, G. Nicholson, Better dispatch application - a success story, Proc. of IEEE/SEMI Advanced Semiconductor Manufacturing Conference and Workshop, pp. 396-399, 1998.

[11]S. C. Huang, J. T. Lin, An interactive scheduler for a wafer probe center in semiconductor manufacturing, International Journal of Production Research, vol. 36, no. 7, pp. $1883-1900,1998$

[12]K.C. Jeong, Y.D. Kim, A real-time scheduling mechanism for a flexible manufacturing system: using simulation and dispatching rules, International Journal of Production Research, vol. 36, no. 9, pp. 2609 2626, 1998.

[13]I. Sabuncuoglu, A study of scheduling rules of flexible manufacturing systems: a simulation approach, International Journal of Production Research, vol. 36, no. 2, pp. $527-546,1998$.

[14]Maria Terasa Costa, Jose Soeiro Ferreira, A simulation analysis of sequencing rules in a flexible flowline, European Journal of Operational Research, vol. 119, pp. 440 - 450, 1999.

[15]O.O. Balogun, K. Popplewell, Towards the integration of flexible manufacturing system scheduling, International Journal of Production Research, vol. 37, no. 15, pp. 3399 - 3428, 1999.

[16]K. H. Hsu, C. C. Lan, Photolithography area dispatching scheme for advanced technology in foundry fabs, Proc. of Semiconductor Manufacturing Technology Workshop, pp. $211-216,2000$.

[17]Bo-Wei Hsieh, Chun-Hung Chen, Shi-Chung Chang, Scheduling semiconductor wafer fabrication by using ordinal optimization-based simulation, IEEE Trans. on Robotics and Automation, vol. 17, no. 5, pp. $599-608,2001$

[18]Yeong-Dae Kim, Jae-Gon Kim, Bum Choi, Hyung-Un Kim, "Production scheduling in a semiconductor wafer fabrication facility producing multiple product types with distinct due dates, IEEE Trans. on Robotics and Automation, vol. 17, no. 5, pp. 589-598, 2001.

[19]Ah-Chih Huang, Li-Chen Fu, Ming-Hung Lin, Shun-Yu Lin, Modeling, scheduling, and prediction for wafer fabrication: queueing colored Petri-net and GA based approach, Proc. of IEEE International Conference on Robotics and Automation, vol. 3, pp. 3187 - 3192, 2002.

[20]Russ M. Dabbas, John W. Fowler, A new scheduling approach using combined dispatching criteria in wafer fabs, IEEE Trans. on Semiconductor Manufacturing, vol. 16, no. 3, pp. 501 - 510, 2003.

[21]Tsung-Che Chiang, Li-Chen Fu, Solving the FMS scheduling problem by critical ratio-based heuristics and the genetic, Proc. of IEEE Conference on Robotics and Automation, vol. 3, pp. 3131-3136, 2004. 